\title{
Multi Attribute Monitoring of antibody charge variants by cation-exchange chromatography coupled to native mass spectrometry
}

Markus Haberger ${ }^{\mathbf{a}}$, Anna-Katharina Heidenreich ${ }^{\mathbf{a}}$, Michaela Hook ${ }^{\mathbf{a}}$, Jürgen

Fichtl $^{\text {a }}$, Rainer Langa, Florian Cymer ${ }^{\mathrm{b}}$, Mahdi Adibzadeh ${ }^{\mathrm{b}}$, Felix Kuhne ${ }^{\mathrm{a}}$, Harald Wegele $^{\mathbf{a}}$, Dietmar Reusch ${ }^{\mathbf{a}}$, Lea Bonnington ${ }^{\mathbf{a}}$, and Patrick Bulau ${ }^{\mathbf{a}}$ *

a Pharma Technical Development, Roche Diagnostics GmbH, 82377 Penzberg, Germany

b Pharma Technical Development, F. Hoffmann-La Roche Ltd, c, 4070 Basel,

Switzerland

* Corresponding author:

Patrick Bulau

mailto: patrick.bulau@roche.com 
Table S1: Summary of analytical data from CEC peak fractionation showing assignments, relative abundances and main variants observed

\begin{tabular}{|c|c|c|c|c|c|c|c|}
\hline Test Method & Product attribute & RM & AP3 & AP2 & AP1 & MP & BP \\
\hline \multirow{24}{*}{$\begin{array}{l}\text { LC-MS tryptic } \\
\text { peptide } \\
\text { mapping }\end{array}$} & HC1 wild-type & 81.5 & 20.9 & 20.6 & 92.2 & 92.0 & 80.1 \\
\hline & HC1-N-338 deam & 2.4 & 47.3 & 4.7 & n.d. & n.d. & n.q. \\
\hline & HC1-N-341-deam & 11.1 & 15.5 & 54.4 & 4.5 & 4.8 & n.q. \\
\hline & HC1-N-338-suc & $<0.5$ & 1.4 & $<0.5$ & n.d. & n.d. & $<0.5$ \\
\hline & HC1-N-341-suc & 0.4 & 0.5 & 2.0 & $<0.5$ & $<0.5$ & $<0.5$ \\
\hline & HC1-N-338-G0F-N & 0.6 & 0.6 & 4.1 & $<0.5$ & $<0.5$ & 0.6 \\
\hline & HC1-N-338-G0F & 1.3 & 1.1 & 9.5 & $<0.5$ & $<0.5$ & 1.1 \\
\hline & HC1-N-338-G1F & $<0.5$ & 1.0 & 2.0 & $<0.5$ & $<0.5$ & $<0.5$ \\
\hline & HC1-N-338-G2F & $<0.5$ & 0.9 & 0.6 & $<0.5$ & $<0.5$ & $<0.5$ \\
\hline & HC1-N-338-G1S1F & $<0.5$ & 2.7 & $<0.5$ & $<0.5$ & $<0.5$ & $<0.5$ \\
\hline & HC1-N-338-G2S1F & $<0.5$ & 3.3 & $<0.5$ & $<0.5$ & $<0.5$ & $<0.5$ \\
\hline & HC1-N-338-G2S2F & $<0.5$ & $<0.5$ & $<0.5$ & $<0.5$ & $<0.5$ & $<0.5$ \\
\hline & $\mathrm{HC} 1 / \mathrm{HC} 2-\mathrm{N}-163-\mathrm{G} 1 \mathrm{~S} 1 \mathrm{~F}$ & $<0.1$ & $<0.1$ & $<0.1$ & 0.4 & $<0.1$ & $<0.1$ \\
\hline & $\begin{array}{c}\text { HC1-S-230-Xyl-Gal-Gal- } \\
\text { GlcA-GlcNAc }\end{array}$ & $<0.5$ & $<0.5$ & $<0.5$ & 2.2 & $<0.5$ & $<0.5$ \\
\hline & $\begin{array}{c}\text { HC1-S-230-Xyl-Gal-Gal- } \\
\text { GlcA }\end{array}$ & $<0.5$ & $<0.5$ & $<0.5$ & 3.3 & $<0.5$ & $<0.5$ \\
\hline & $\begin{array}{c}\text { HC1-S-230-Xyl-Gal- } \\
\text { Neu5Ac }\end{array}$ & $<0.5$ & $<0.5$ & $<0.5$ & 1.4 & $<0.5$ & $<0.5$ \\
\hline & HC1-S-230-Xyl-Gal & $<0.5$ & $<0.5$ & $<0.5$ & 0.5 & $<0.5$ & $<0.5$ \\
\hline & HC-C-terminal K & 0.7 & 1.0 & 1.1 & 1.1 & 1.3 & 2.0 \\
\hline & $\begin{array}{l}\text { HC-C-terminal P } \\
\text { Amidation }\end{array}$ & 4.8 & 5.3 & 4.3 & 4.7 & 3.2 & 6.4 \\
\hline & LC-N-terminal-pyE & 100.0 & 100.0 & 100.0 & 100.0 & 100.0 & 100.0 \\
\hline & HC-N-terminal-pyE & 100.0 & 99.9 & 99.9 & 100.0 & 99.9 & 100.0 \\
\hline & $\begin{array}{l}\text { LC1-K-55- Glycated } \\
\text { (Trypsin digestion) }\end{array}$ & $<0.5$ & $<0.5$ & $<0.5$ & 1.3 & $<0.5$ & $<0.5$ \\
\hline & $\begin{array}{c}\text { LC1-K-55-Glycated } \\
\text { (Thermolysin digestion) }\end{array}$ & $<0.5$ & $<0.5$ & $<0.5$ & 7.0 & $<0.5$ & $<0.5$ \\
\hline & $\begin{array}{l}\text { HC-Fab-N-Glycosylation } \\
\text { (main variant) }\end{array}$ & GOF/GOF/GOF & G0F/G1F/G1S1F & G0F/G0F/G0F & G0F/G0F/G1S1F & G0F/G0F/GOF & n.d. \\
\hline
\end{tabular}




\begin{tabular}{|c|c|c|c|c|c|c|c|}
\hline Test Method & Product attribute & RM & AP3 & AP2 & AP1 & MP & BP \\
\hline \multirow{6}{*}{$\begin{array}{c}\text { Native SEC-UV- } \\
\text { MS }\end{array}$} & HC-Fab-N-Glycosylation & 3.4 & 12.9 & 25.2 & 6.2 & 0.6 & n.d. \\
\hline & $\begin{array}{c}\text { HC-GS4 Linker-O- } \\
\text { Glycosylation } \\
\text { (main variant) }\end{array}$ & n.d. & n.d. & $\begin{array}{c}\text { G0F/G0F, } \\
\text { Xyl-[PO3]-Gal- } \\
\text { Gal-GlcA }\end{array}$ & $\begin{array}{c}\text { GOF/GOF, } \\
\text { Xyl-Gal-Gal-GlcA }\end{array}$ & n.d. & n.d. \\
\hline & $\begin{array}{l}\text { HC-GS4 Linker-O- } \\
\text { Glycosylation }\end{array}$ & n.d. & n.d. & 3.3 & 30.5 & n.d. & n.d. \\
\hline & $\begin{array}{l}\text { HC-Fc-N-Glycosylation } \\
\text { (main variant) }\end{array}$ & G0F/G0F & G0F/G0F & G0F/G0F & G0F/G1F & GOF/GOF & GOF/GOF \\
\hline & $\begin{array}{l}\text { SEC-MS HC-Fc-N- } \\
\text { Glycosylation only }\end{array}$ & 94.7 & 87.1 & 71.5 & 63.4 & 99.4 & 67.0 \\
\hline & Fc-glycosylation only & G0F/G0F & G0F/G0F & G0F/G0F & G0F/G0F & GOF/GOF & GOF/GOF \\
\hline \multirow{3}{*}{$\begin{array}{c}\text { FabALACTICA } ® \\
\text { (IgdE) } \\
\text { Digest }\end{array}$} & Fab N-glycosylation only & GOF & G2S1F & GOF & G1S1F & GOF & not tested \\
\hline & Fab glycation only & 21.4 & - & - & 30.5 & 15.1 & not tested \\
\hline & Fab O-Xylose only & 6.7 & 16.1 & 11.9 & 41.7 & 5.9 & not tested \\
\hline $\begin{array}{l}\text { Functional } \\
\text { testing }\end{array}$ & Cell based potency & 100 & 3 & 48 & 124 & 117 & 98 \\
\hline
\end{tabular}

HC heavy chain; LC light chain; N asparagine; K lysine; P proline; N- and O-glycosylation (see Figure 1); deam deamidation; suc succinimide; PO3 phosphite; n.d. not detected; n.q. not quantifiable (due to high aggregate/fragment content) 
Supporting Information

Table S2: SEC-UV-MS analysis of CEC fractions and online CEC-MS analysis (Figures 3 and 6): Peak assignment according to accurate mass determination at charge state +26

\begin{tabular}{|c|c|c|c|}
\hline $\begin{array}{c}\text { Peak } \\
\text { Number/Name }\end{array}$ & $\begin{array}{c}\text { Experimental mass } \\
\text { value }\end{array}$ & $\begin{array}{c}\text { Theoretical mass } \\
\text { value }\end{array}$ & $\begin{array}{c}\text { Proposed TCB Mab } \\
\text { charge variant }\end{array}$ \\
\hline 1 & 197664 & 197663 & $\begin{array}{c}\text { HC1-S230-Xyl-Gal-Gal- } \\
\text { GlcA }\end{array}$ \\
\hline 2 & 197867 & 197864 & $\begin{array}{c}\text { HC1-S230-Xyl-Gal-Gal- } \\
\text { GlcA-GlcNAc }\end{array}$ \\
\hline 3 & 197988 & 198002 & HC1-S230-Xyl-Gal-Gal- \\
GlcA
\end{tabular}


Table S3: Amino acid sequence and detected c fragment ions (EThcD fragmentation) of a peptic TCB Mab peptide (AP3 fraction) containing HC-N338/341.

\begin{tabular}{|c|c|}
\hline $\begin{array}{c}\text { c-ion } \\
\text { (detected } \boldsymbol{m} / \mathbf{z})\end{array}$ & $\begin{array}{c}\text { Amino acid sequence } \\
\text { (HC1 position number) }\end{array}$ \\
\hline c1 (181.097) & Y (331) \\
\hline c2 (344.160) & Y (332) \\
\hline c3 (505.175) & C (333) \\
\hline c4 (604.244) & V (334) \\
\hline c5 (760.345) & R (335) \\
\hline c6 (897.404) & $\mathrm{H} \mathrm{(336)}$ \\
\hline c7 (954.425) & $\mathrm{G} \mathrm{(337)}$ \\
\hline c8 (n.d.) & $\mathrm{N} \mathrm{(338)}$ \\
\hline c9 (n.d.) & $\mathrm{F} \mathrm{(339)}$ \\
\hline c10 (n.d.) & $\mathrm{G} \mathrm{(340)}$ \\
\hline c11 (n.d.) & $\mathrm{N} \mathrm{(341)}$ \\
\hline c12 (n.d.) & $\mathrm{S} \mathrm{(342)}$ \\
\hline c8 N-glycosylated & N-glycosylation type \\
\hline 1271.547 & $\mathrm{~N}$ \\
\hline 1417.605 & $\mathrm{~N}+\mathrm{F}$ \\
\hline 1474.627 & $\mathrm{~N}+\mathrm{N}$ \\
\hline 1620.685 & $\mathrm{~N}+\mathrm{N}+\mathrm{F}$ \\
\hline 1636.679 & $\mathrm{~N}+\mathrm{N}+\mathrm{M}$ \\
\hline 1782.738 & $\mathrm{~N}+\mathrm{N}+\mathrm{F}+\mathrm{M}$ \\
\hline
\end{tabular}

n.d. $=$ not detected

$\mathrm{N}=\mathrm{N}$-acetylglucosamine

$\mathrm{F}=$ Fucose

$\mathrm{M}=$ Mannose 
Supporting Information

Figure S1: SEC-UV-MS analysis of TCB Mab charge variant fractions (UV chromatographic trace)

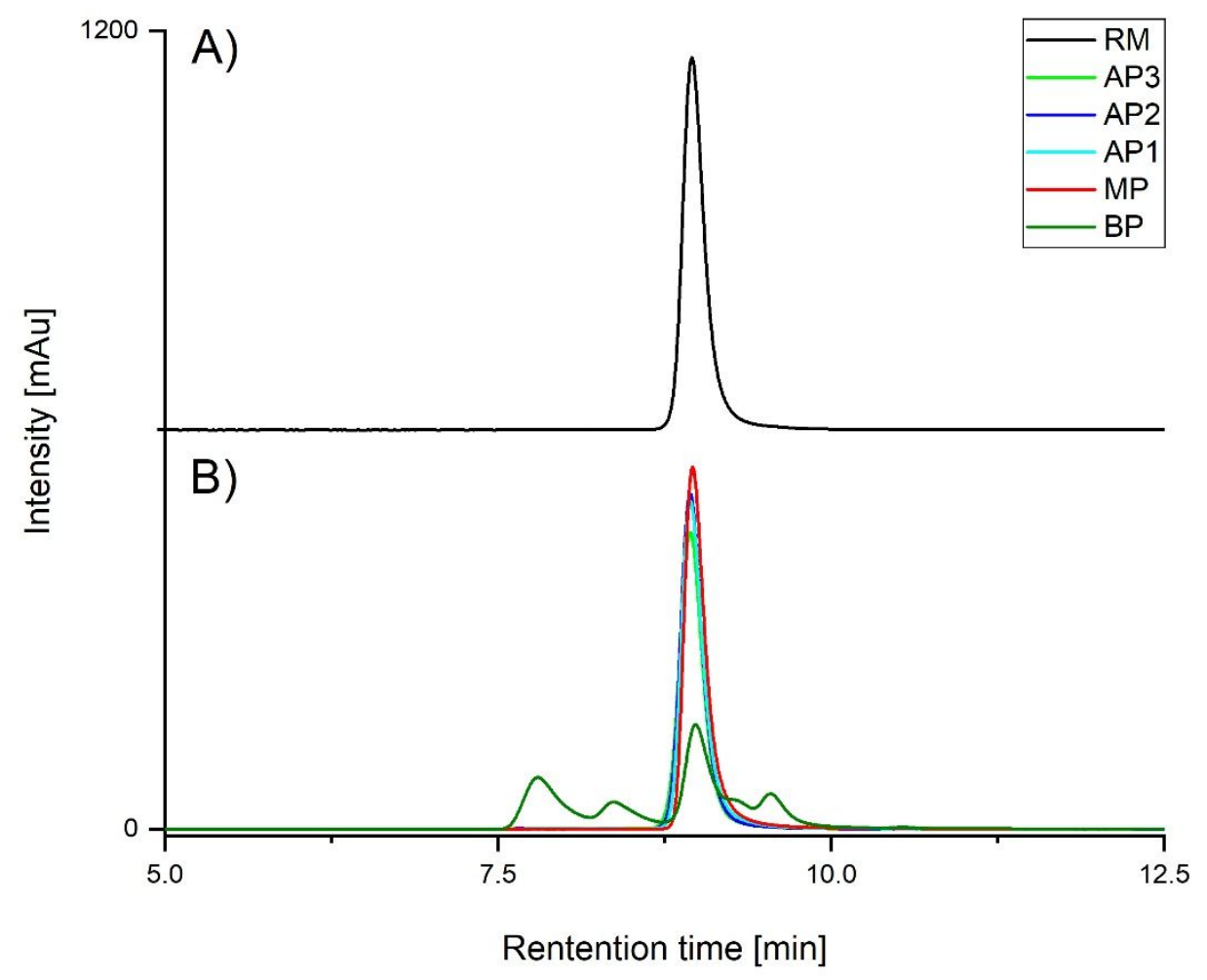

